\title{
Damage Control can Prevent Unreversed Brain Injury in Trauma Patients
}

\section{Fade Ahmad Alhussien}

MRCS Ireland, Syrian Board, Specialist in Saudi Arabia, Bani Malik General Hospital, KSA

*Corresponding Author: Fade Ahmad Alhussien, MRCS Ireland, Syrian Board, Specialist in Saudi Arabia, Bani Malik General Hospital, KSA.

Received: July 02, 2019; Published: July 19, 2019

DOI: 10.31080/ASNE.2019.02.0086

Sever haemorrage is a common cause of disability following traumatic ruptures of large vessels and parenchymal organs. The long -term functional outcome is largely determined by the degree of brain damage resulting from shock associated cerebral blood flow. When cardiac arrest occurs, it is essential to start cardiopulmonary resuscitation within two minutes. After three minutes, global cerebral ischenia can lead to progressively worsening brain damage, By nine minutes, severe and irrvesible brain damage is likely. It is important to notice that subcortical but not cortical perfusion is maintained during hypovolymic shock. Maintenance of subcortical perfusion is dependent on a1-AR signals in the brain. As a result, some patients live in vegetative state. main body of article, on 15 /5/2019 across -border infiltrator had been brought to emergency department complaining of gun shoot in his left gluteal muscles. The anatomical inlet in gluteal but the track towards abdomen, injured the gluteal muscles, pelvic bone, retroperitoneal muscles, and abdominal viscera, without outlet. His symptoms was thirsty, abdominal pain, and disability to move left lower limb. On clinical examination, he was lethargic confused, pallor, tachypnic, but with patent airway and acceptable air entry to the lung, additionally he was unsatble, critically ill, the blood pressure was undetectable, the preferal pulses were not palpable, only carotid pulse, which mean blood pressure between 60-70mmHG. And all this signs mean that patient in hypovolymic shock grade four, with dangerous of multi organ failure, especially brain. Up to advanced trauma life support protocol the treatment was simultaneously resuscitation and bleeding control. Blood transfusion, plasma, fluids, were started, but the difficult problem was no on call anaesthesia specialist, which mean routinely to refer the patient to nearest hospital, which is one hour far away. The patient was unstable instead of resuscitation, and started to loss his consciousness, furthermore in blood gases analyses acidosis was present. As a result, it is impossible to transfer the patient to another hospital, and the final out come will be cardiac arrest. My decision was to make damage control, and i will be the person who give anaesthesia and surgeon in same time. I shifted the patient to operation room, and he was given medazolam $7 \mathrm{mg}$, ketamine $30 \mathrm{mg}$,atracurium $25 \mathrm{mg}$, my emergency resident worked as ventilator for me, laprotomy was done, free blood itra abdomen, huge retroperitoneal haemorrhage were identified, so packing was done, and bleeding was controlled. Gradually blood pressure improved, reach to $80 / 50$ additionally refilling time and urine out put improved. And finally patient became in border of stability in vital sign, on ventilator, with empty drain. At that moment i decided to transfer to icu as second stage of damage control, and later on reexplanation. And exactly this what happened, ct and ivf FFP, blood were given, and re-explorated to repair bowel injuries, finally patient was extubated, admitted in male word, and my patient is still alive.

\section{Conclusion}

Haemorrhage will cause unreversed brain injury if untreated before cardiac arrest. Surgeon can give anaethesia to survive truama patient in special circumstances. Damage control, which mean a treatment strategy to stop bleeding in truama patient, is golden option. The indication for damage control is hypothermia less than 35c, acidosis PH less than 7,2, coagulopathy, long operation more than 90 minutes, and hymodynamic unstability mean blood pressure less than 70 MMHG. Surgeon will control haemrrhage, give blood, FFB, platelets, and decrease fluids, and later on re-exploration.

\section{Volume 2 Issue 8 August 2019 C) All rights are reserved by Fade Ahmad Alhussien.}

